\title{
Influencia del proceso de fibrilación y desfibrilación enzimática en las propiedades mecánicas de hilos de fibras celulósicas regeneradas obtenidas por el proceso NMMO
}

\author{
F. Carrillo*, J. Valldeperas ${ }^{* *}$, D. Evans ${ }^{* * *}$, M. Huson $^{* * *}$ y X. Colom
}

Resumen La obtención de fibras hiladas a partir de una solución de celulosa en $\mathrm{N}$-óxido de $\mathrm{N}$ metilmorfolina hidratado permite obtener fibras de celulosa regenerada, con excelentes propiedades mecánicas por medio de un proceso industrial no contaminante (proceso NMMO). Sin embargo, estas fibras presentan una propiedad especial, su elevada fibrilación, principalmente durante los tratamientos en húmedo, que es posible controlar y explotar mediante las etapas de fibrilación mecánica y desfibrilación enzimática de los productos fabricados. En el presente trabajo se estudiaron las propiedades mecánicas (deformación a la rotura, módulo de Young, resistencia máxima a la tracción y tenacidad) de muestras de hilo de esta nueva fibra, evaluando la influencia de los tratamientos industriales de fibrilación mecánica y desfibrilación enzimática. Se observó que la tenacidad y la resistencia a la rotura de las muestras disminuyen significativamente después de estos tratamientos y, principalmente, durante el tratamiento enzimático. Por este motivo, el control de estas operaciones es un factor clave para asegurar unas propiedades mecánicas aceptables de los productos fabricados.

Palabras clave Lyocell. Fibrilación. Enzimas. Propiedades mecánicas.

\section{Effects of fibrillation and enzymatic defibrillation treatments on mechanical properties of regenerated cellulosic yarns from nmmo process}

\begin{abstract}
Fibre spinning from a solution of cellulose in $\mathrm{N}$-methyl-morpholine- $\mathrm{N}$-oxidehydrate is an environmentally friendly process (NMMO), which yields cellulose fibres with excellent mechanical properties. A special feature of these fibbers is their ability to fibrillate in the swollen state. It is possible to control and exploit fibrillation of this kind of fibre (generic name: lyocell) through mechanical fibrillation and enzymatic defibrillation treatments. The mechanical properties (elongation at break, Young's modulus, tensile strenght and tenacity) of solvent (NMMO)-spun lyocell yarns samples have been studied in untreated condition as well as after industrial mechanical fibrillation and enzymatic defibrillation treatments. It is observed that the tenacity of lyocell yarn decreases greatly as a result of the treatments, particularly after the enzymatic treatment. For this reason, the control of these operations plays a critical role in ensuring acceptable mechanical properties of the yarns.
\end{abstract}

Keywords

Lyocell. Fibrillation. Enzymes. Mechanical Properties.

\section{INTRODUCCIÓN}

Con el creciente énfasis en la minimización de las emisiones contaminantes, se han realizado numerosos esfuerzos con objeto de encontrar un sistema alternativo al uso del sulfuro de carbono para la producción de fibras de celulosa regenerada (visco-

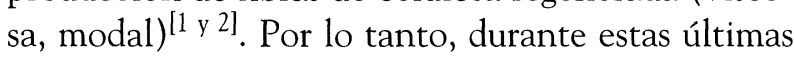
décadas ha habido un gran interés científico y tecnológico en el desarrollo de sistemas no contaminantes basados en el uso de disolventes no acuosos de la celulosa ${ }^{[2-4]}$, entre los que podemos destacar

(*) Departament d'Enginyeria Química, EUETIT - UPC, Colom 1, Terrassa, E-08222, Barcelona (España).

$\left({ }^{* *}\right)$ INTEXTER - UPC, Colom 15, Terrassa, E-08222, Barcelona (España).

$\left.{ }^{* * *}\right)$ CSIRO Textile and Fibre Technology, Belmont, VIC 3216, (Australia). 
algunos realmente prometedores como el $\mathrm{N}$-óxido de $\mathrm{N}$-metilmorfolina $\left(\mathrm{NMMO} / \mathrm{H}_{2} \mathrm{O}\right)^{[5 \text { y } 6]}$, dimetilformamida/tetraóxido de dinitrógeno (DMF/ $\left.\mathrm{N}_{2} \mathrm{O}_{4}\right)^{[2]}$, y el $\mathrm{N}, \mathrm{N}$-dimetilacetamida/cloruro de litio $(\mathrm{DMAc} / \mathrm{LiCl})^{[3]}$. Actualmente, sólo el proceso $\mathrm{NMMO}^{[7]}$ ofrece una alternativa, comercialmente viable, entre los diferentes métodos de producción propuestos. El producto obtenido, reconocido con el nombre genérico de lyocell ${ }^{[8 \text { y }}{ }^{9]}$ (la denominación abreviada es $\operatorname{CLY}^{[10]}$ ), ha aparecido en el mercado con diferentes denominaciones comerciales: Tencel $^{\circledR}$ (Courtaulds) $^{[11]}$, Lenzing Lyocell ${ }^{\circledR}$ (Lenzing), Newcell ${ }^{\circledR}$ (Azko Faser) y Alceru ${ }^{\circledR}$ (Titk Rudolstadt/Schwarza).

Las ventajas de esta nueva fibra celulósica frente a las fibras convencionales de viscosa radican en que, el proceso de producción es menos contaminante ${ }^{[12]}$ y el producto obtenido presenta mejores propiedades mecánicas: alto módulo y tenacidad, principalmente, en húmedo ${ }^{[13]}$. Sin embargo, presenta una propiedad especial, su alta fibrilación durante los tratamientos en húmedo, es decir, la aparición de fibrillas a lo largo de la superficie de la fibra, que debe ser controlada con la incorporación de etapas previas de tratamiento que consisten en una fibrilación primaria, mediante acción mecánica controlada, seguida de una posterior desfibrilación enzimática. Por este motivo, es importante la selección de unas condiciones óptimas de tratamiento, con objeto de conseguir los efectos deseados sin una degradación excesiva de sus propiedades mecánicas.

En el presente estudio se analiza el efecto de las etapas de fibrilación y desfibrilación enzimática sobre las propiedades mecánicas (módulo de Young, deformación a la rotura, resistencia máxima a la tracción y tenacidad) de muestras de lyocell.

\section{METODOLOGÍA}

Se utilizaron muestras de tejido de Lyocell de Courtaulds, con denominación comercial Tencel ${ }^{\circledR}$, de $283 \mathrm{~g} / \mathrm{m}^{2}$ de masa superficial.

Las muestras de tejido se sometieron industrialmente al proceso de fibrilación por acción mecánica en una máquina Jet, durante $90 \mathrm{~min}$, a la temperatura de $100^{\circ} \mathrm{C}$, con una relación de baño 1:10 y en medio alcalino $\left(\mathrm{Na}_{2} \mathrm{CO}_{3}\right)$ a $\mathrm{pH}$ 10. Posteriormente, se realizó la desfibrilación enzimática, con enzimas de celulasa, con una concentración de enzima del $3 \%$ s.p.f., a la temperatura de $55^{\circ} \mathrm{C}$ y pH controlado de 5,5, durante un tiempo de $90 \mathrm{~min}$. Finalmente se desactivó el complejo enzimático por incremento de la temperatura hasta $85^{\circ} \mathrm{C}$, manteniéndola durante $10 \mathrm{~min}$.

Para la determinación experimental de las curvas esfuerzo/deformación se prepararon probetas de hilo, a partir de las muestras de tejido, de 50 mm de longitud y acondicionadas a $21^{\circ} \mathrm{C}$ y $65 \%$ de humedad relativa durante $24 \mathrm{~h}$.

La caracterización de las propiedades mecánicas se realizó, utilizando una máquina Instron 4501. La velocidad de extensión ha sido de $10 \mathrm{~mm} / \mathrm{min}$ y la fuerza aplicada de $100 \mathrm{~N}$, bajo condiciones de humedad y temperatura constantes, $65 \%$ y $21^{\circ} \mathrm{C}$, respectivamente. El ensayo se repitió 5 veces para cada una de las probetas preparadas determinando la curva esfuerzo/deformación. A partir de estas curvas se calcularon las propiedades mecánicas de tracción, expresando el resultado como valor medio de las cinco medidas.

\section{RESULTADOS Y DISCUSIÓN}

En la figura 1 se representan las curvas esfuerzo (N/tex)/deformación (\%) promedio obtenidas, para cada una de las muestras analizadas. A partir de estas curvas se calcularon las principales propiedades mecánicas de tracción: módulo de Young, deformación a la rotura, resistencia máxima a la tracción y tenacidad, cuyos valores promedio se resumen en la tabla I.

En las figuras 1 y 2 puede apreciarse cómo los tratamientos efectuados sobre el sustrato modifican significativamente las propiedades mecánicas de tracción en el punto de rotura, disminuyendo los valores de deformación y esfuerzo a rotura y, por lo tanto, la tenacidad, mientras que no se observan cambios apreciables del módulo de Young.

Los resultados obtenidos demuestran que la acción de ambos tratamientos provocan una disminución de las propiedades mecánicas del sustrato, debido a un proceso de degradación a nivel macro y microestructural. Durante el tratamiento de fibrilación, la acción mecánica produce una rápida aparición de fibrillas a lo largo de la superficie de la fibra que modifican su aspecto superficial. Esta acción de abrasión sobre la fibra posibilita el desprendimiento de algunas de estas fibrillas superficiales hacia el baño de tratamiento, reduciéndose así la densidad lineal de las muestras (Tabla I). Por lo tanto, la fibrilación reduce el número de fibrillas por unidad de sección que podrán soportar la carga de tracción, lo que comporta una disminución significativa del esfuerzo a rotura $(4,6 \%)$ y de la deformación máxima (15,4\%). 
Influencia del proceso de fibrilación y desfibrilación enzimática...

Tabla I. Propiedades mecánicas de tracción de las muestras

Table I. Mechanical properties of the samples

\begin{tabular}{|c|c|c|c|c|c|c|}
\hline \multirow[b]{2}{*}{ Muestra } & \multicolumn{6}{|c|}{ Propiedades mecánicas } \\
\hline & Estadística & $\begin{array}{c}\text { Densidad } \\
\text { lineal (tex) }\end{array}$ & $\begin{array}{l}\text { Módulo de } \\
\text { Young } \\
\text { (N/tex) }\end{array}$ & $\begin{array}{l}\text { Deformación } \\
\text { a rotura }(\%)\end{array}$ & $\begin{array}{c}\text { Esfuerzo a } \\
\text { la rotura } \\
(\mathrm{N} / \mathrm{tex})\end{array}$ & $\begin{array}{c}\text { Tenacidad } \\
(\mathrm{J} / \mathrm{g})\end{array}$ \\
\hline \multirow{3}{*}{ A } & Media & 56,80 & 1,79 & 12,54 & 0,175 & 12,13 \\
\hline & Desv. Estándar & 3,35 & 0,0862 & 0,8882 & 0,0128 & 1,5879 \\
\hline & C.V. (\%) & 5,89 & 4,8 & 7,1 & 7,3 & 13,1 \\
\hline \multirow{3}{*}{ B } & Media & 52,00 & 1,80 & 10,61 & 0,167 & 9,34 \\
\hline & Desv. Estándar & 6,78 & 0,0977 & 0,6858 & 0,0113 & 1,0707 \\
\hline & C.V. $(\%)$ & 13,04 & 5,4 & 6,5 & 6,7 & 11,5 \\
\hline \multirow{3}{*}{ C } & Media & 53,20 & 1,86 & 7,71 & 0,121 & 5,05 \\
\hline & Desv. Estándar & 5,76 & 0,1340 & 0,4988 & 0,0063 & 0,4540 \\
\hline & C.V. $(\%)$ & 10,83 & 7,2 & 6,5 & 5,2 & 9 \\
\hline
\end{tabular}

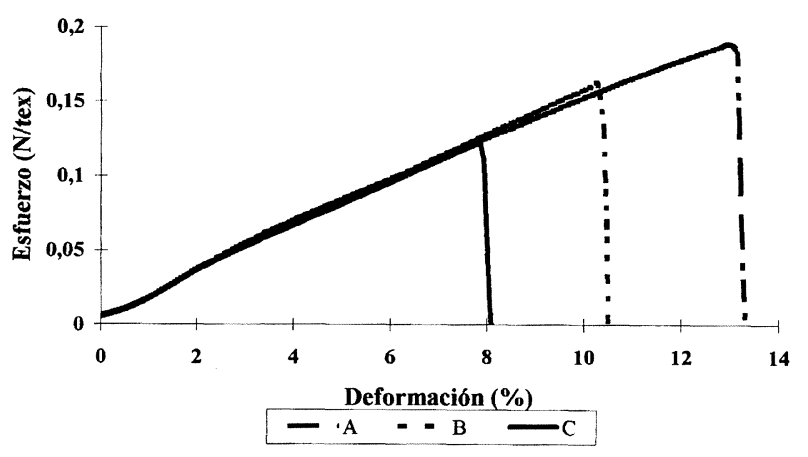

Figura 1. Comparación de las curvas esfuerzo/deformación para las tres muestras de estudio. (A:Muestra original, B: Fibrilada, C: Desfibrilación enzimática).

Figure 1. Specific stress-strain curves of lyocell yams. (Original A, Fibrillated B, Enzymatic C).

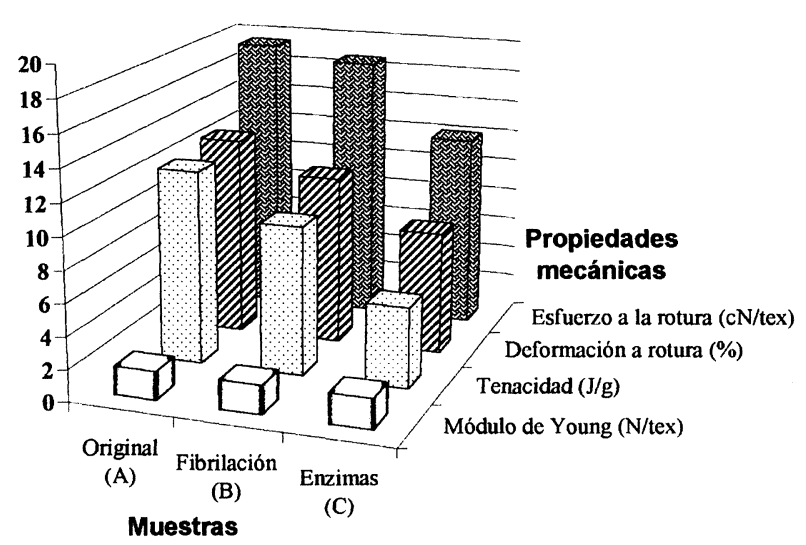

Figura 2. Efecto de los tratamientos sobre las propiedades mecánicas de tracción.

Figure 2. Effect of the treatments on the mechanical properties.
Durante el tratamiento enzimático, mediante la acción sinérgica de los componentes que constituyen el enzima de celulasa, se produce la hidrólisis específica del enlace $\beta-1,4$ - glicosídico de las macromoléculas que componen el polímero de celulosa $^{[14 \text { y } 15]}$. Este ataque se produce, preferentemente, en las zonas amorfas, produciéndose un acortamiento de las cadenas del polímero, formándose cadenas de celulosa más pequeñas, que se transforman en glucosa cuando la hidrólisis es completa. Como consecuencia de esta acción hidrolítica, se observa una disminución importante de la tenacidad manteniéndose prácticamente constante el módulo de Young (Fig.1).

En la figura 3 se representa el porcentaje de disminución parcial y total que se produce en las propiedades mecánicas de tracción como consecuencia de los tratamientos aplicados.

Como se puede observar en la figura 3 , ambos tratamientos influyen directamente sobre las propiedades mecánicas del sustrato. No obstante, el proceso de degradación enzimática es mayor, reduciendo los valores de deformación y esfuerzo a rotura un 27,3 y $27,5 \%$ respectivamente, frente a una reducción del 15,4 y 4,6\% durante la fibrilación. Esta diferencia de comportamiento es debida a que la acción degradativa del proceso de fibrilación provoca la rotura física de las fibrillas de las fibras y, por lo tanto, la degradación se produce a nivel macroestructural. Por el contrario, la hidrólisis enzimática provoca una degradación microestructural en la que se ven afectadas directamente las macromoléculas de celulosa.

Rev. Metal. Madrid 37 (2001) 


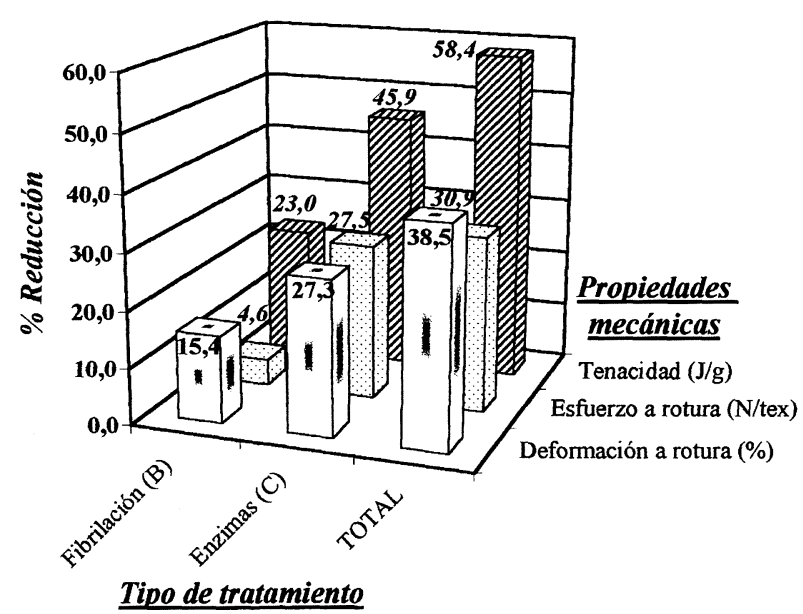

Figura 3. Reducción de las propiedades mecánicas.

Figure 3. Decrease (\%) of the mechanical properties.

Por otra parte, se debe destacar que el tratamiento mecánico de fibrilación tiene mayor influencia sobre la deformación que sobre el esfuerzo a rotura, mientras que el tratamiento enzimático produce una reducción similar de ambas propiedades mecánicas ( $27 \%$ aproximadamente).

La acción conjunta de los dos tratamientos estudiados produce una reducción total de la tenacidad de las muestras del 58,4 \%, indicando nuevamente el elevado efecto degradativo de los mismos. Por lo tanto, a partir de la combinación de ambos tratamientos, es necesario llegar a un compromiso que permita conseguir el efecto final deseado sin degradar excesivamente sus propiedades mecánicas, teniendo en cuenta que la acción específica del enzima las puede reducir considerablemente.

\section{CONCLUSIONES}

Los pretratamientos de fibrilación y desfibrilación enzimática necesarios para el control del fenómeno de fibrilación de las materias fabricadas con hilos de fibras lyocell, modifican significativamente sus propiedades mecánicas de tracción. Principalmente, se produce una disminución importante de la tenacidad, como consecuencia de una disminución de la deformación y esfuerzo a rotura, sin observarse cambios significativos del módulo de Young.

Esta reducción de las propiedades mecánicas a rotura es consecuencia directa de la acción degra- dativa de ambos tratamientos, ya que el tratamiento mecánico produce cambios macroestructurales, fundamentalmente por abrasión superficial del sustrato y rotura de fibrillas mientras que, por otra parte, el tratamiento enzimático degrada las macromoléculas del polímero por escisión hidrolítica química del enlace glicosídico.

Asimismo, también se ha podido comprobar, que la degradación es más intensa durante el tratamiento con enzimas, debido a la hidrólisis específica que se produce, por lo que el control de esta operación es fundamental para conseguir los efectos deseados sin degradar excesivamente las excelentes propiedades mecánicas de estas nuevas fibras celulósicas regeneradas.

\section{Agradecimientos}

Los autores desean expresar su agradecimiento a Nicole Phair por su ayuda en el desarrollo experimental, y a la organización IFACTT por su financiación económica.

\section{REFERENCIAS}

[1] P.A. KoCH y F. LyOCell, Chem. Fibres Int. 47 (1997) 298 303.

[2] A.S. Chegolya, D.D. Grinshpan y E.Z. Burd, Text. Res. J. 59 (1989) 501-506.

[3] K.H. Young, H.C. Kyung y S.L. Wha, Text. Res. J. 68 (1998) 65-69.

[4] S.P. PAPKOV, Fibre Chem. 28 (1996) 1-5.

[5] C.C. McCorsley y J.K. Varga, U.S. Patent 4142913, 1979.

[6] A.M. Bochek, G.A. Petropavlovsky y A.V. YAKImAnsky, Cellulose and Cellulose derivates: PhysicoChemical Aspects and Industrial Applications, Woodhead Publishing Limited, Abington Hall, Abington, 1995.

[7] P. Weigel y P. Fink, Lenzinger Berichte 76 (1997) 115-119.

[8] N.N. Lyocell, Chem. Fibres Int. 45 (1995) 72-30.

[9] ISO 2076, Textiles- Man-made Fibers - Generic names (1995) 12-12, Reference number ISO/TC 38/SC N 1631.

[10] G. Schnegelsberg, Chem. Fibers Int. 46 (1996) 390.

[11] J.M. Taylor y A.L. Harnden, Int. Dyer, Text. Printer, Bleacher Finish. 182 (8) (1997) 14-17.

[12] Marini, Firgo, Eibl, Lenzing Lyocell, Lenzinger Berichte 9 (1994) 53-56.

[13] K. Macfarlane, Chem. Fibers Int. 47 (1997) 328-333.

[14] L.P. Wa.LKER y D.B. WILSON, Bioresource Technol. 36 (1991) 3-14.

[15] B. Nidetzky y W. Steiner, Biotechnol. Bioeng 42 (1993) 469-479. 